\title{
Vibrational dressing in kinetically constrained Rydberg spin systems
}

\author{
Paolo P. Mazza, ${ }^{1}$ Richard Schmidt, ${ }^{2,3}$ and Igor Lesanovsky ${ }^{1,4}$ \\ ${ }^{1}$ Institut für Theoretische Physik, University of Tübingen, \\ Auf der Morgenstelle 14, 72076 Tübingen, Germany \\ ${ }^{2}$ Max-Planck-Institute of Quantum Optics, Hans-Kopfermann-Strasse, 1, 85748 Garching, Germany \\ ${ }^{3}$ Munich Center for Quantum Science and Technology (MCQST), Schellingstr. 4, 80799 München, Germany \\ ${ }^{4}$ School of Physics and Astronomy and Centre for the Mathematics \\ and Theoretical Physics of Quantum Non-Equilibrium Systems, \\ The University of Nottingham, Nottingham, NGr 2RD, United Kingdom
}

(Dated: June 25, 2020)

\begin{abstract}
Quantum spin systems with kinetic constraints have become paradigmatic for exploring collective dynamical behaviour in many-body systems. Here we discuss a facilitated spin system which is inspired by recent progress in the realization of Rydberg quantum simulators. This platform allows to control and investigate the interplay between facilitation dynamics and the coupling of spin degrees of freedom to lattice vibrations. Developing a minimal model, we show that this leads to the formation of polaronic quasiparticle excitations which are formed by many-body spin states dressed by phonons. We investigate in detail the properties of these quasiparticles, such as their dispersion relation, effective mass and the quasiparticle weight. Rydberg lattice quantum simulators are particularly suited for studying this phonon-dressed kinetically constrained dynamics as their exaggerated length scales permit the site-resolved monitoring of spin and phonon degrees of freedom.
\end{abstract}

Introduction.- The precise control and manipulation of quantum systems is of utmost importance both in fundamental physics and for applications in quantum technologies. The last decade has seen an immense effort in the improvement of experimental techniques which enable the exploration of quantum many-body systems [1, 2]. Rydberg atoms are notably suitable for this scope due to their versatility in simulating many-body models [3-5]. In particular, they provide an ideal platform for the realization of spin systems, with applications ranging from quantum information processing [6] to the exploration of fundamental questions concerning thermalization in quantum mechanics [7-9].

Recently, there has been a growing interest in the study of quantum systems in the presence of kinetic constraints, that impose restrictions on the connectivity between many-body configurations. In particular, it has been observed how constraints, which prevent the system from fully exploring the Hilbert space, can lead to peculiar dynamics and an unexpected lack of thermalization even in systems without explicit symmetries [10-23]. A condensed matter manifestation of such effect can be found e.g. in linear $\mathrm{SrCo}_{2} \mathrm{~V}_{2} \mathrm{O}_{8}$ crystal that is described by a spin- $\frac{1}{2} \mathrm{XXZ}$ antiferromagnetic Hamiltonian with a staggered magnetic field [24]. Facilitation is a specific instance of a constrained dynamics. The concept was introduced by Fredrickson and Andersen [25] in the study of kinetic aspects of the glass transition using spin models [26]. Here the excitation of one spin enhances the excitation probability of a neighboring spin. In Rydberg gases such dynamical behavior occurs naturally in the so-called anti-blockade regime [2731 , and the emerging many-body effects have been investigated in detail in many recent works $[32,33]$. Among the studied phenomena are nucleation and growth [34- (a)

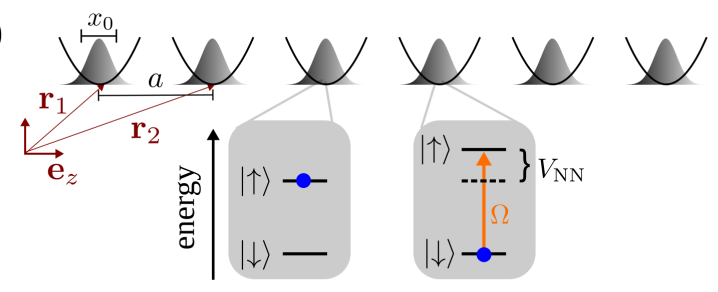

(b)

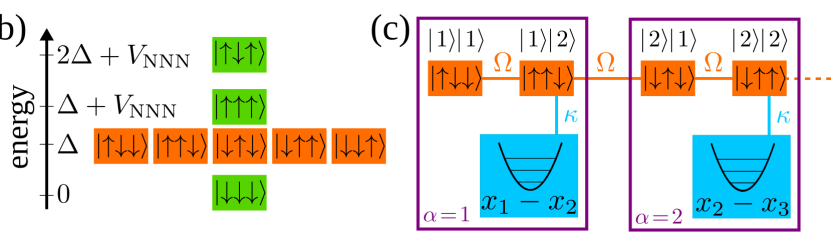

Figure 1: Setting: (a) Schematic representation of the Rydberg quantum simulator. The internal degrees of freedom of each trapped atom are approximated by a two-level system. Here, $\Omega$ is the Rabi frequency of the excitation laser that is detuned from the atomic transition by an energy $\Delta$, and the interaction between nearest neighbors is parameterized by $V_{\mathrm{NN}}$. The atoms are confined in a one-dimensional chain of harmonic traps, where $a$ is the lattice spacing and $x_{0}$ represents the harmonic oscillator length. (b) Hilbert space representation in the facilitation regime. The many-body configurations at energy $\Delta$ are resonant and effectively decoupled from the rest of the Hilbert space. $V_{\mathrm{NNN}}$ is the interaction among next nearest neighbours in Rydberg states. (c) Hilbert space defined by the effective lattice of resonant many-body states and their coupling to harmonic oscillator modes. Only states with two contiguous Rydberg excitations are coupled to the (relative) vibrational mode of the traps.

38], non-equilibrium phase transitions [39-43] as well as Anderson [32, 44] and many-body localization [45].

In this work we are interested in exploring the interplay between facilitated spin excitations and vibrational 
degrees of freedom. Such a scenario naturally occurs in Rydberg lattice quantum simulators [3], where individual atoms are held in oscillator potentials [see Fig. 1(a)], and coupling between spin and vibrations is caused by state-dependent mechanical forces [46, 47]. We develop a minimal model that describes the emerging complex many-body dynamics and permits a perturbative expansion in the spin-phonon coupling strength. The dressing of the spin dynamics through lattice vibrations leads to the formation of a polaronic quasiparticle [48] for which we analyse the dispersion relation, the effective mass and the $Z$-factor, determining the quasiparticle weight. The perturbative results are compared with numerical simulations. Using Rydberg quantum simulators for exploring this physics is particularly appealing as these platforms allow the probing of spin and vibrational degrees of freedom. Thus, using side-band spectroscopy [49], the phonon cloud that dresses the spin excitation should be directly observable in experiments.

Facilitated Rydberg lattice.- We consider a chain of $N$ traps (e.g. optical tweezers) [50, 51] each loaded with a single Rydberg atom (see Fig. 1). The Rydberg atoms can be effectively described as a two-level system in which $|\downarrow\rangle_{i}$ represents an atom in the ground state in the $\mathrm{i}-t h$ trap and $|\uparrow\rangle_{i}$ an atom in the Rydberg state. The Hamiltonian of the system is

$$
H=\sum_{i=1}^{N}\left(\frac{\Omega}{2} \hat{\sigma}_{i}^{x}+\Delta \hat{n}_{i}+\sum_{j \neq i} V\left(\mathbf{r}_{i}, \mathbf{r}_{j}\right) \hat{n}_{i} \hat{n}_{j}+\omega a_{i}^{\dagger} a_{i}\right)
$$

where $i, j$ are indices that label the lattice sites, $\Omega$ is the Rabi frequency, and $\Delta$ is the detuning of the Rydberg excitation laser from the single atom resonance. The interactions among Rydberg states are parameterized by the potential $V\left(\mathbf{r}_{i}, \mathbf{r}_{j}\right)$ which may be, for example, of van-der-Waals or dipolar type. Furthermore, we have introduced the spin operators $\hat{\sigma}_{i}^{x}=|\uparrow\rangle_{i}\langle\downarrow|+| \downarrow\rangle_{i}\langle\uparrow|$, $\hat{n}_{i}=|\uparrow\rangle_{i}\langle\uparrow|$. The interaction potential depends on the atomic positions $\mathbf{r}_{j}=\mathbf{r}_{j}^{(0)}+\delta \mathbf{r}_{j}$ where the coordinate of the centre of the $j$-th trap is given by $\mathbf{r}_{j}^{(0)}=a j \mathbf{e}_{z}$, with $a$ the lattice constant, c.f. Fig. 1. The fluctuations $\delta_{j}=\frac{\delta r_{j}}{a}$ around the trap center can be expressed in terms of the bosonic operators (obeying $\left.\left[a_{i}, a_{j}^{\dagger}\right]=\delta_{i, j}\right)$ as $\delta_{j}=\frac{x_{0}}{a}\left(a_{j}^{\dagger}+\right.$ $\left.a_{j}\right)$, where $x_{0}=\sqrt{\hbar /(m \omega)}$ is the harmonic oscillator length and $m$ the atomic mass. Assuming that $\delta r_{i} \ll a$, i.e. the interparticle separation is much larger than the fluctuations around the equilibrium positions, we can expand the interaction potential to first order obtaining a coupling term between the Rydberg excitations and the vibrational trap modes. Here we are considering only the longitudinal modes because, as shown in the Supplemental material, in one-dimensional lattices the coupling with the transverse modes is negligible at the first order of the perturbative expansion of the potential. In this case we obtain:

$$
V\left(\mathbf{r}_{i}, \mathbf{r}_{j}\right) \simeq V\left(\mathbf{r}_{i}^{0}, \mathbf{r}_{j}^{0}\right)+G\left(\mathbf{r}_{i}^{0}, \mathbf{r}_{j}^{0}\right)\left(\delta_{i}-\delta_{j}\right)
$$

Here, $G\left(\mathbf{r}_{i}^{0}, \mathbf{r}_{j}^{0}\right)$ is the gradient of the potential [59].

In the facilitation regime, the interaction between two neighboring atoms is cancelled by the laser detuning, $\Delta=-V\left(\mathbf{r}_{i}^{0}, \mathbf{r}_{i+1}^{0}\right)$. This means that transitions between many-body configurations of the type $|\downarrow \uparrow \downarrow \downarrow\rangle \leftrightarrow|\downarrow \uparrow \uparrow \downarrow\rangle$ become resonant [see Fig. 1(b)]. In order to simplify the dynamics further we assume that the interaction between next-nearest-neighbours is larger than the Rabi frequency, i.e. $V\left(\mathbf{r}_{i}^{0}, \mathbf{r}_{i+2}^{0}\right) \gg|\Omega|$. This prevents the growth of clusters and constrains the evolution of a single initial seed atom to a subspace in which at most two adjacent atoms are excited, e.g. $|\uparrow \downarrow \downarrow \downarrow\rangle \leftrightarrow|\uparrow \uparrow \downarrow \downarrow\rangle \leftrightarrow|\downarrow \uparrow \downarrow \downarrow\rangle \leftrightarrow$ $|\downarrow \uparrow \uparrow \downarrow\rangle \leftrightarrow \ldots$, as shown in Fig. 1(b).

This subspace of many-body states defines an effective one-dimensional lattice with a two-site unit cell, for which we introduce the labels $|\alpha\rangle|s\rangle$. Here, the variable $\alpha$ denotes the position of the leftmost excited spin and $s$ the number of excited spins, i.e., for $\alpha=1,|\alpha\rangle|1\rangle=|\uparrow \downarrow \downarrow \downarrow\rangle$, $|\alpha\rangle|2\rangle=|\uparrow \uparrow \downarrow \downarrow\rangle$ and $|\alpha+1\rangle|1\rangle=|\downarrow \uparrow \downarrow \downarrow\rangle$, etc. [see Fig. 1(c)]. On this effective lattice, the Hamiltonian (1) can be rewritten as

$$
\begin{aligned}
H= & \Omega \sum_{\alpha}\left(|\alpha\rangle\left\langle\alpha\left|\mu^{x}+\mu^{-}\right| \alpha+1\right\rangle\langle\alpha|+\text { h.c. }\right) \\
& +\kappa \sum_{\alpha} \frac{\mu^{z}-\mathbb{1}}{2}|\alpha\rangle\langle\alpha|\left(a_{\alpha+1}^{\dagger}+a_{\alpha+1}-a_{\alpha}^{\dagger}-a_{\alpha}\right) \\
& +\omega \sum_{\alpha} a_{\alpha}^{\dagger} a_{\alpha}
\end{aligned}
$$

where $\mu^{x}=|1\rangle\langle 2|+| 2\rangle\left\langle 1\left|, \quad \mu^{-}=\right| 1\right\rangle\langle 2|$ and $\mu^{z}=|1\rangle\langle 1|-| 2\rangle\langle 2|$ are spin operators, that characterize the two non-equivalent types of sites in the lattice and $\kappa=-\sqrt{\frac{1}{2 m \omega}} G\left(\mathbf{r}_{i}^{0}, \mathbf{r}_{j}^{0}\right)$.

Vibrational dressing.- Through Eq. (3) it is evident that bosons, which correspond to the trap vibrations, interact only with states where $s=2$, i.e., with states in which there are two adjacent excited spins, as shown in Fig. 1(c). In order to simplify the description we rewrite the Hamiltonian (3) by introducing the Fourier transformed bosonic modes $a_{j}=\frac{1}{\sqrt{N}} \sum_{p=-N / 2}^{N / 2} \mathrm{e}^{\mathrm{i} \frac{2 \pi}{N} j p} A_{p}$, which yields

$$
\begin{aligned}
H= & \Omega \sum_{\alpha}|\alpha\rangle\langle\alpha| \mu^{x}+\Omega \sum_{\alpha}\left(\mu^{-}|\alpha+1\rangle\langle\alpha|+\text { h.c. }\right) \\
& +\frac{\kappa\left(\mu^{z}-\mathbb{1}\right)}{2 \sqrt{N}} \sum_{p}\left[\left(\mathrm{e}^{-\mathrm{i} \frac{2 \pi}{N} p}-1\right) \mathrm{e}^{-\mathrm{i} \frac{2 \pi}{N} \hat{\alpha}} A_{p}^{\dagger}+\text { h.c. }\right] \\
& +\omega \sum_{p} A_{p}^{\dagger} A_{p} .
\end{aligned}
$$


where $\hat{\alpha}=\sum_{\alpha} \alpha|\alpha\rangle\langle\alpha|$ denotes the lattice position operator. We can decouple the lattice from the bosonic modes using the Lee-Low-Pines transformation [52]

$$
U=\exp \left[-\mathrm{i} \hat{\alpha} \sum_{p} \frac{2 \pi p}{N} A_{p}^{\dagger} A_{p}\right] .
$$

Introducing the Fourier modes of the quasi-particles, $|\alpha\rangle=\frac{1}{\sqrt{N}} \sum_{q=-N / 2}^{N / 2} \mathrm{e}^{\mathrm{i} \frac{2 \pi}{N} q}|q\rangle$, the transformed Hamiltonian reads $U^{\dagger} H U=\sum_{q}|q\rangle\langle q| H_{q}$, with

$$
\begin{aligned}
H_{q}= & \Omega\left[\mu^{+}\left(1+\mathrm{e}^{-\mathrm{i} \frac{2 \pi}{N}\left(\sum_{p} p A_{p}^{\dagger} A_{p}+q\right)}\right)+\text { h.c. }\right] \\
& +\frac{\kappa\left(\mu^{z}-\mathbb{1}\right)}{\sqrt{2 N}} \sum_{p}\left[\left(\mathrm{e}^{-\mathrm{i} \frac{2 \pi}{N} p}-1\right) A_{p}^{\dagger}+\text { h.c. }\right] \\
& +\omega \sum_{p} A_{p}^{\dagger} A_{p} .
\end{aligned}
$$

By virtue of the canonical transformation the quasiparticle momentum $q$ is now a conserved quantum number, which simplifies tremendously the subsequent analysis. Further manipulations, which are detailed in the Supplemental Material, allow us to finally obtain

$$
\begin{aligned}
H_{q}= & \omega \sum_{p} \tilde{A}_{p}^{\dagger} \tilde{A}_{p} \mathbb{1}-\Omega \cos \left[\frac{\pi}{N}\left(\sum_{p} p A_{p}^{\dagger} A_{p}+q\right)\right] \mu^{z} \\
& +H_{\mathrm{int}}^{\mathrm{I}}+H_{\mathrm{int}}^{\mathrm{II}}
\end{aligned}
$$

with $H_{\mathrm{int}}^{\mathrm{I}}=-\frac{\kappa^{2}}{\omega}\left(\mathbb{1}-\mu^{x}\right)$ and the displaced bosonic operators $\tilde{A}_{p}=A_{p}+\frac{\kappa}{\omega \sqrt{N}}\left(\mathrm{e}^{-\mathrm{i} \frac{2 \pi}{N} p}-1\right)$. An explicit expression for $H_{\mathrm{int}}^{\mathrm{II}}$ is given in the Supplemental Material. Note, that despite the achieved simplification, the Hamiltonian (7) is highly non-trivial and now describes many-body spin states coupled to a bath of interacting phonons.

To investigate the vibrational dressing of the facilitation dynamics we first consider the decoupling limit $\kappa=0$. In this case the spectrum of Hamiltonian (7) is given by bands that appear in pairs with positive and negative curvature [see Fig. 2(a)]. There are infinitely many pairs, forming a ladder with a spacing given by the trap frequency $\omega$. The ground state band has the tight-binding dispersion relation, i.e. $E_{\mathrm{GS}}=-\Omega \cos \left(\frac{\pi}{N} q\right)$. Note that in the limit of $N \gg 1$, the argument of the cosine becomes a continuous variable $-\pi \leq \frac{2 \pi}{N} q \leq \pi$. In the following we assume for simplicity that the trap (phonon) frequency is larger than the twice the laser Rabi frequency, $\omega>$ $2 \Omega$. In this case the ground state band is well separated from the remaining ones. Crucially, this regime is within reach of current technology from an experimental point of view. In fact, in order to be able to observe coherent dynamics, we must have $\omega>2 \Omega \gg \gamma$ with $\gamma$ being the decay rate of the Rydberg atoms. Typically, $\gamma \approx$ $10^{4} \mathrm{~Hz}$ and frequencies larger than $10^{5} \mathrm{~Hz}$ can be achieved (a)

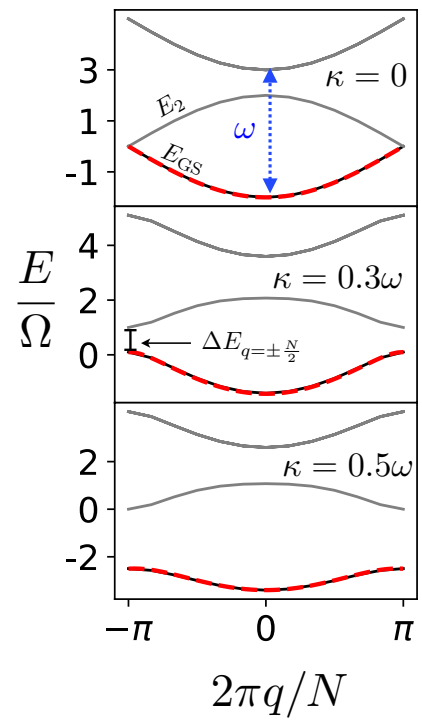

(b)

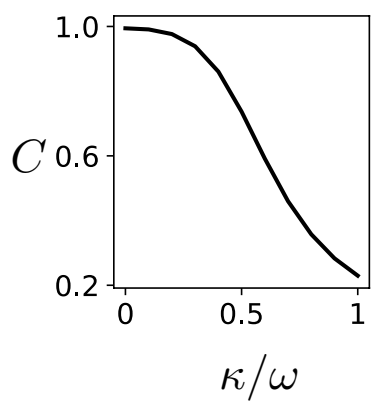

Numerics $=-n^{--n}$ Analytics
Figure 2: Band Structure: (a) Comparison between the analytical form Eq. (8) and numerical calculations obtained for $N=12$ and truncation of the maximum number of phonons per site to 3 . The non-interacting bands form a ladder with spacing $\omega$. As the interaction between the lattice and phonons increases, the band degeneracies at the edges are lifted and the ground state band is flattened. (b) Curvature $C$ of the groundstate band computed for quasiparticle momentum $q=0$, numerical results.

experimentally, for both $\Omega[44]$ and $\omega$ [49]. Furthermore, both the Rydberg and the ground state ought to be trapped as demonstrated in Ref. [53].

In the presence of interactions between the propagating Rydberg excitation and the phonons, i.e. for $\kappa \geq 0$, the energy bands, defining the spectrum of Eq. (7), are modified. In particular, we observe the lifting of the degeneracy of the ground state and the first excited band at the band edges together with a flattening of the band structure. The decrease of the band curvature, shown in Fig. 2(b), is a consequence of the phonon-dressing of the spin excitation which leads to the formation of a polaron quasiparticle which is characterized by a correspondingly increased effective band mass. In order to obtain a qualitative understanding of the observed renormalization of the band structure we adopt a perturbative approach to the solution of Eq. (7). The term $H_{\text {int }}^{\mathrm{I}}$ couples states with quasiparticle momentum $q$ of the ground state band, $\left|\Psi_{q}^{(0)}\right\rangle$, to the first excited band. At first order in perturbation theory, this correction can be computed solving a two-level eigenvalue problem for each $q$. We have also an additional correction to the energy given by the action of $H_{\mathrm{int}}^{\mathrm{II}}$ GS. This yields the dressed value for the ground 

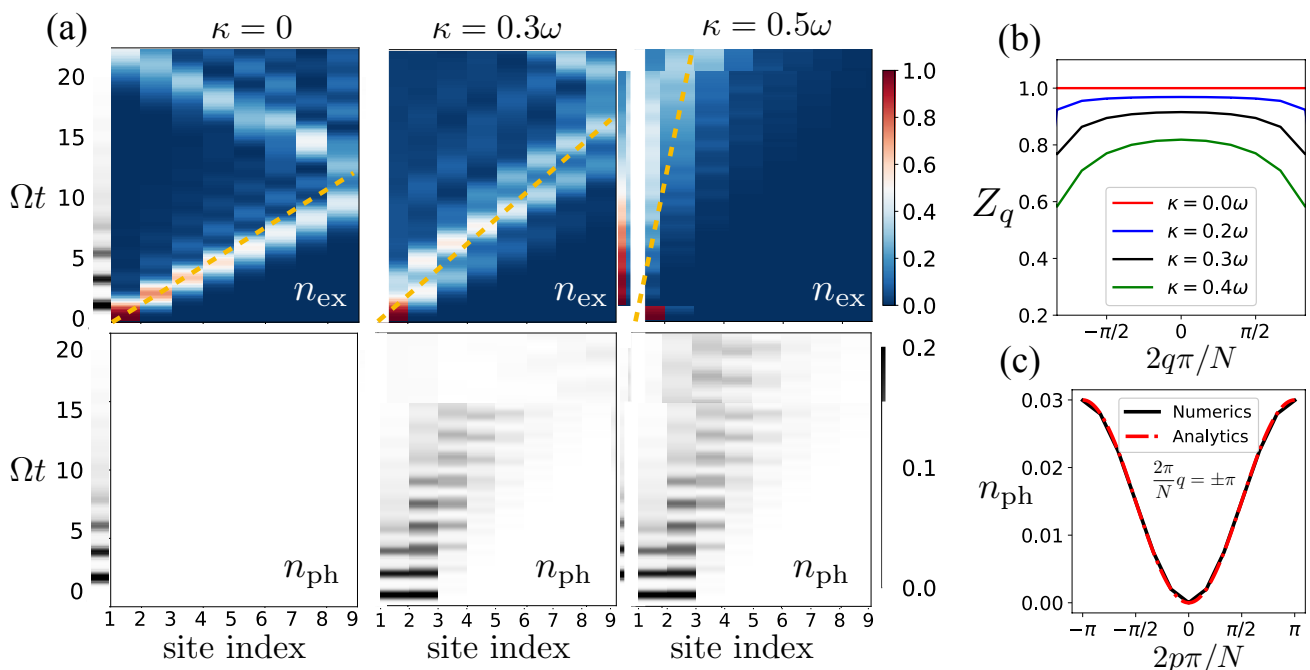

Figure 3: Polaron dynamics: (a) Density plot of the Rydberg and phonon excitation for different values of the interaction strength $\kappa / \omega$. The first (second) row shows the Rydberg (phonon) density. For $\kappa=0$, i.e. in absence of interactions, a ballistic spreading of the Rydberg excitation is observed and no phonons are generated (orange dashed lines are a guide to the eye). At $\kappa=0.3 \omega(\kappa=1.5 \Omega$ and $\omega=5 \Omega)$ the propagation of the Rydberg excitation is slowed down until it almost comes to a halt (on the timescale shown) when $\kappa=0.5 \omega$. (b) Momentum-dependent $Z$-factor for different values of the coupling strength. (c) Occupation number of the phonon modes in momentum space (labelled by $p$ ) of the ground state for quasi-particle momentum $\frac{2 \pi}{N} q= \pm \pi$ and $\kappa=0.3 \Omega$. We compare the analytical result of Eq. (9) with numerical data obtained using exact diagonalization on a system up to $N=12$ sites.

state band, $E_{\mathrm{GS}}^{(1)}(q)$ :

$E_{\mathrm{GS}}^{(1)}=-\sqrt{\Omega^{2} \cos ^{2}\left(\frac{\pi q}{N}\right)+\frac{\kappa^{4}}{\omega^{2}}}-\frac{\kappa^{2}}{\omega}+\frac{\Omega \kappa^{2}}{\omega^{2} N} \xi_{N} \cos \left(\frac{\pi q}{N}\right)$,

with $\xi_{N}=\frac{4 \cos \left(\frac{\pi}{N}\right) \cot \left(\frac{\pi}{2 N}\right) \cos \left(\frac{\pi q}{N}\right)}{2 \cos \left(\frac{\pi}{N}\right)+1}$. As it can be seen in Fig. 2, there is good agreement between the analytical result and the numerics.

Dressed facilitation dynamics.- The interaction between the Rydberg atoms and the phonons that leads to the phonon dressing and corresponding band flattening, results in a slowdown of propagating facilitated Rydberg excitations. This effect is shown in Fig. 3(a), where we display the real-time dynamics of both Rydberg excitations and phonons. For the simulations we performed exact diagonalization on a system of size $N=10$ and we truncated the local bosonic Hilbert space allowing a maximum number of three bosons per site. The initial state contains a single Rydberg excitation at the left edge of the lattice and no bosons, i.e. $|\uparrow \downarrow \downarrow \ldots\rangle \otimes|0,0,0 \ldots\rangle$. Consequently, such wave packet states of the form $\left|\psi_{\text {in }}\right\rangle=|\ldots \uparrow \downarrow \downarrow \ldots\rangle$ in real space correspond to superpositions of momentum states that live on the first two excited bands due to a mixing between the states introduced in the diagonalization of the Hamiltonian (6).

The data in Fig. 3(a) shows that the stronger the coupling $\kappa$ the more pronounced becomes the phonon trail that is carried and left behind by the propagating Rydberg excitation. In Rydberg quantum simulator experiments it is standard to measure the Rydberg density [3]. It is, however, also possible to determine the local phonon density by side-band spectroscopy, as demonstrated in Ref. [49]. Remarkably, this makes it possible to use Rydberg quantum simulators to directly detect and map out the phonon cloud in-situ and in real-time, which remains elusive in solid state systems and most ultracold atom platforms.

The magnitude of the phonon dressing can be quantified by the Z-factor which is defined by the overlap of the dressed polaron state $\left|\bar{\psi}_{q}\right\rangle$ with its non-interacting counterpart $\left|\psi_{q}^{(0)}\right\rangle, Z_{q}=\left|\left\langle\psi_{q}^{(0)} \mid \bar{\psi}_{q}\right\rangle\right|^{2}[48,54,55]$. The calculation of the $Z$-factor from exact diagonalization, see Fig. 3(b) shows that, although the phonon dressing is strong, still a well-defined polaron quasiparticle exists.

We also compute the phonon occupation number in momentum space in the dressed ground state $\left|\bar{\psi}_{q}\right\rangle$, i.e. $n_{\mathrm{ph}}(p, q)$, with $p$ and $q$ being the phonon and quasi-particle momentum respectively, $n_{\mathrm{ph}}(p, q)=$ $\left\langle\bar{\psi}_{q}\left|A_{p}^{\dagger} A_{p}\right| \bar{\psi}_{q}\right\rangle$. While this quantity cannot be computed exactly analytically, at first non-zero order one finds:

$$
\begin{aligned}
n_{\mathrm{ph}}^{(1)}(q, p) & =\frac{\kappa^{2}}{\omega^{2} N}\left\langle\psi_{q}^{(0)}\left|\left(\mathrm{e}^{\mathrm{i} \frac{2 \pi}{N} p}-1\right)\left(\mathrm{e}^{-\mathrm{i} \frac{2 \pi}{N} p}-1\right)\right| \psi_{q}^{(0)}\right\rangle \\
& =2 \frac{\kappa^{2}}{\omega^{2} N}\left[1-\cos \left(\frac{2 \pi}{N} p\right)\right]
\end{aligned}
$$

where $-\pi \leq \frac{2 \pi}{N} p \leq \pi$. Note, that this result does not depend on the quasiparticle momentum $q$. Such a depen- 
dence enters at higher order in perturbation theory and leads to a $q$-dependent coefficient to Eq. (9). In fact our numerical calculations confirm a dependence of the form

$$
n_{\mathrm{ph}}(p, q)=2 \frac{\kappa^{2}}{\omega^{2} N} C^{*}(q)\left[1-\cos \left(\frac{2 \pi}{N} p\right)\right]
$$

with a numerically determined coefficient $C^{*}(q)$. In Fig. 3(c) which we show the phonon occupation number $n_{\mathrm{ph}}(p, q)$ at the edges of the ground state band, $\frac{2 \pi}{N} q= \pm \pi$, at $\kappa=0.3 \Omega$. The agreement between numerical and analytical results from Eq. (9) is excellent.

Conclusions.- We have shown how the non-equilibrium dynamics of a facilitated Rydberg atoms chain is dramatically affected by interactions with trap vibrations. This coupling leads to a dressing of the propagating excitations and shows the emergence of a slow-dynamics induced by a flattening of the quasi-particle bands. The latter can be interpreted as a polaronic effect that leads to an increase of the effective mass. The phonon dressing, as discussed here, might have links to other timely research questions: it was recently pointed out, that lattice Hamiltonians coupled to bosons can offer a possible setup for the observation of fractons [56], which are currently much studied in the context of ergodicity breaking in quantum systems. Moreover, tuning the interaction between the excitations and the phonons permits to control the spreading of information within the system, which is a timely theme in the domain of quantum technology $[57,58]$.

Acknowledgements.- We acknowledge discussions with C. Groß, W. Li and F. Gambetta. I.L. and R.S. acknowledge support from the DFG through SPP 1929 (GiRyd). I.L. is acknowledging support by the "WissenschaftlerRückkehrprogramm GSO/CZS" of the Carl-Zeiss-Stiftung and the German Scholars Organization e.V. R. S. is supported by the Deutsche Forschungsgemeinschaft (DFG, German Research Foundation) under Germany's Excellence Strategy - EXC-2111 - project ID 390814868.

[1] I. Bloch, J. Dalibard, and W. Zwerger, Rev. Mod. Phys. 80, 885 (2008), URL https://link.aps.org/doi/10. 1103/RevModPhys.80.885.

[2] R. E. Wooten, B. Yan, and C. H. Greene, Phys. Rev. B 95, 035150 (2017), URL https://link.aps.org/doi/10. 1103/PhysRevB.95.035150.

[3] A. Browaeys and T. Lahaye, Nat. Phys. 16, 132-142 (2020).

[4] M. T. Eiles and C. H. Greene, Phys. Rev. A 95, 042515 (2017), URL https://link.aps.org/doi/10. 1103/PhysRevA.95.042515.

[5] K. Singer, J. Stanojevic, M. Weidemüller, and R. Côté, Journal of Physics B: Atomic, Molecular and Optical Physics 38, S295 (2005), URL https://doi.org/10. 1088\%2F0953-4075\%2F38\%2F2\%2F021.
[6] M. Saffman, T. G. Walker, and K. Mølmer, Rev. Mod. Phys. 82, 2313 (2010), URL https://link.aps.org/ doi/10.1103/RevModPhys.82.2313.

[7] A. Polkovnikov, K. Sengupta, A. Silva, and M. Vengalattore, Rev. Mod. Phys. 83, 863 (2011).

[8] L. D'Alessio, Y. Kafri, A. Polkovnikov, and M. Rigol, Advances in Physics 65, 239 (2016), https://doi.org/10.1080/00018732.2016.1198134, URL https://doi.org/10.1080/00018732.2016.1198134.

[9] P. Calabrese, F. H. L. Essler, and G. Mussardo, Journal of Statistical Mechanics: Theory and Experiment 2016, 064001 (2016), URL https://doi.org/10.1088\% $2 \mathrm{~F} 1742-5468 \% 2 \mathrm{~F} 2016 \% 2 \mathrm{~F} 06 \% 2 \mathrm{~F} 064001$.

[10] C. Ates, J. P. Garrahan, and I. Lesanovsky, Phys. Rev. Lett. 108, 110603 (2012), URL https://link.aps.org/ doi/10.1103/PhysRevLett.108.110603.

[11] Z. Lan, M. van Horssen, S. Powell, and J. P. Garrahan, Phys. Rev. Lett. 121, 040603 (2018), URL https://link. aps.org/doi/10.1103/PhysRevLett.121.040603.

[12] C. J. Turner, A. A. Michailidis, D. A. Abanin, M. Serbyn, and Z. Papic, Nature Physics 14, 745 (2018), ISSN 1745-2481, URL https://doi.org/10. 1038/s41567-018-0137-5.

[13] S. Choi, C. J. Turner, H. Pichler, W. W. Ho, A. A. Michailidis, Z. Papić, M. Serbyn, M. D. Lukin, and D. A. Abanin, preprint arXiv:1812.05561 (2018).

[14] V. Khemani, C. R. Laumann, and A. Chandran, Phys. Rev. B 99, 161101 (2019), URL https://link.aps.org/ doi/10.1103/PhysRevB.99.161101.

[15] C. J. Turner, A. A. Michailidis, D. A. Abanin, M. Serbyn, and Z. Papić, Phys. Rev. B 98, 155134 (2018).

[16] W. W. Ho, S. Choi, H. Pichler, and M. D. Lukin, Phys. Rev. Lett. 122, 040603 (2019).

[17] M. Kormos, M. Collura, G. Takács, and P. Calabrese, Nature Physics 13, 246 EP (2016), URL https://doi. org/10.1038/nphys3934.

[18] A. Lerose, F. M. Surace, P. P. Mazza, G. Perfetto, M. Collura, and A. Gambassi, arXiv:1911.07877 (2019).

[19] C.-J. Lin and O. I. Motrunich, Phys. Rev. B 97, 144304 (2018), URL https://link.aps.org/doi/10. 1103/PhysRevB.97.144304.

[20] C.-J. Lin and O. I. Motrunich, Phys. Rev. Lett. 122, 173401 (2019), URL https://link.aps.org/doi/10. 1103/PhysRevLett.122.173401.

[21] N. Shiraishi and T. Mori, Phys. Rev. Lett. 119, 030601 (2017), URL https://link.aps.org/doi/10. 1103/PhysRevLett.119.030601.

[22] S. Moudgalya, S. Rachel, B. A. Bernevig, and N. Regnault, Phys. Rev. B 98, 235155 (2018), URL https://link.aps . org/doi/10.1103/PhysRevB.98.235155.

[23] S. Moudgalya, N. Regnault, and B. A. Bernevig, Phys. Rev. B 98, 235156 (2018), URL https://link.aps.org/ doi/10.1103/PhysRevB.98.235156.

[24] Z. Wang, J. Wu, W. Yang, A. K. Bera, D. Kamenskyi, A. N. Islam, S. Xu, J. M. Law, B. Lake, C. Wu, et al., Nature 554, 219 (2018).

[25] G. H. Fredrickson and H. C. Andersen, Phys. Rev. Lett. 53, 1244 (1984), URL https://link.aps.org/doi/10. 1103/PhysRevLett. 53.1244.

[26] J. P. Garrahan and D. Chandler, Phys. Rev. Lett. 89, 035704 (2002), URL https://link.aps.org/doi/10. 1103/PhysRevLett.89.035704.

[27] C. Ates, T. Pohl, T. Pattard, and J. M. Rost, Phys. Rev. Lett. 98, 023002 (2007), URL https://link.aps.org/ 
doi/10.1103/PhysRevLett.98.023002.

[28] T. Amthor, C. Giese, C. S. Hofmann, and M. Weidemüller, Phys. Rev. Lett. 104, 013001 (2010), URL https://link. aps.org/doi/10.1103/PhysRevLett.104.013001.

[29] M. Gärttner, K. P. Heeg, T. Gasenzer, and J. Evers, Phys. Rev. A 88, 043410 (2013), URL https://link.aps.org/ doi/10.1103/PhysRevA.88.043410.

[30] J. T. Young, T. Boulier, E. Magnan, E. A. Goldschmidt, R. M. Wilson, S. L. Rolston, J. V. Porto, and A. V. Gorshkov, Phys. Rev. A 97, 023424 (2018), URL https: //link.aps.org/doi/10.1103/PhysRevA.97.023424.

[31] M. Gärttner, K. P. Heeg, T. Gasenzer, and J. Evers, Phys. Rev. A 88, 043410 (2013), URL https://link.aps.org/ doi/10.1103/PhysRevA.88.043410.

[32] M. Ostmann, M. Marcuzzi, J. Minar, and I. Lesanovsky, Quantum Science and Technology 4, 02LT01 (2019), URL https://doi .org/10.1088\%2F2058-9565\%2Faaf29d.

[33] J. P. Garrahan, Physica A: Statistical Mechanics and its Applications 504, 130 (2018), ISSN 03784371, lecture Notes of the 14th International Summer School on Fundamental Problems in Statistical Physics, URL http://www.sciencedirect.com/ science/article/pii/S0378437117313985.

[34] H. Schempp, G. Günter, M. Robert-de Saint-Vincent, C. S. Hofmann, D. Breyel, A. Komnik, D. W. Schönleber, M. Gärttner, J. Evers, S. Whitlock, et al., Phys. Rev. Lett. 112, 013002 (2014), URL https://link.aps.org/ doi/10.1103/PhysRevLett.112.013002.

[35] I. Lesanovsky and J. P. Garrahan, Phys. Rev. A 90, 011603 (2014), URL https://link.aps.org/doi/10. 1103/PhysRevA.90.011603.

[36] A. Urvoy, F. Ripka, I. Lesanovsky, D. Booth, J. P. Shaffer, T. Pfau, and R. Löw, Phys. Rev. Lett. 114, 203002 (2015), URL https://link.aps.org/doi/10. 1103/PhysRevLett. 114.203002.

[37] M. M. Valado, C. Simonelli, M. D. Hoogerland, I. Lesanovsky, J. P. Garrahan, E. Arimondo, D. Ciampini, and O. Morsch, Phys. Rev. A 93, 040701 (2016), URL https://link.aps.org/doi/10.1103/PhysRevA.93. 040701.

[38] M. Mattioli, A. W. Glätzle, and W. Lechner, New Journal of Physics 17, 113039 (2015), URL https://doi.org/10. 1088\%2F $1367-2630 \% 2$ F $17 \% 2$ F $11 \% 2 F 113039$.

[39] N. Malossi, M. M. Valado, S. Scotto, P. Huillery, P. Pillet, D. Ciampini, E. Arimondo, and O. Morsch, Phys. Rev. Lett. 113, 023006 (2014), URL https://link.aps.org/ doi/10.1103/PhysRevLett.113.023006.

[40] M. Marcuzzi, M. Buchhold, S. Diehl, and I. Lesanovsky, Phys. Rev. Lett. 116, 245701 (2016), URL https ://link. aps.org/doi/10.1103/PhysRevLett.116.245701.

[41] F. Letscher, O. Thomas, T. Niederprüm, M. Fleischhauer, and H. Ott, Phys. Rev. X 7, 021020 (2017), URL https: //link.aps.org/doi/10.1103/PhysRevX.7.021020.

[42] R. Gutiérrez, C. Simonelli, M. Archimi, F. Castellucci, E. Arimondo, D. Ciampini, M. Marcuzzi, I. Lesanovsky, and O. Morsch, Phys. Rev. A 96, 041602 (2017), URL https://link.aps.org/doi/10. 1103/PhysRevA.96.041602.

[43] S. Helmrich, A. Arias, G. Lochead, T. M. Wintermantel, M. Buchhold, S. Diehl, and S. Whitlock, Nature 577, 481 (2020), URL https://doi.org/10.1038/ s41586-019-1908-6.

[44] M. Marcuzzi, J. Minar, D. Barredo, S. de Léséleuc, H. Labuhn, T. Lahaye, A. Browaeys, E. Levi, and
I. Lesanovsky, Phys. Rev. Lett. 118, 063606 (2017), URL https://link.aps.org/doi/10.1103/PhysRevLett. 118.063606 .

[45] M. Ostmann, M. Marcuzzi, J. P. Garrahan, and I. Lesanovsky, Phys. Rev. A 99, 060101 (2019), URL https://link.aps.org/doi/10.1103/PhysRevA.99. 060101.

[46] R. Belyansky, J. T. Young, P. Bienias, Z. Eldredge, A. M. Kaufman, P. Zoller, and A. V. Gorshkov, Phys. Rev. Lett. 123, 213603 (2019), URL https://link.aps.org/doi/ 10.1103/PhysRevLett.123.213603.

[47] F. M. Gambetta, W. Li, F. Schmidt-Kaler, and I. Lesanovsky, Phys. Rev. Lett. 124, 043402 (2020), URL https://link.aps.org/doi/10.1103/PhysRevLett. 124.043402 .

[48] A. S. Alexandrov and N. F. Mott, Polarons and Bipolarons (WORLD SCIENTIFIC, 1996), URL https://doi.org/ $10.1142 / 2784$.

[49] A. M. Kaufman, B. J. Lester, and C. A. Regal, Phys. Rev. X 2, 041014 (2012), URL https://link.aps.org/doi/ 10.1103/PhysRevX.2.041014.

[50] H. Bernien, S. Schwartz, A. Keesling, H. Levine, A. Omran, H. Pichler, S. Choi, A. S. Zibrov, M. Endres, M. Greiner, et al., Nature 551, 579 (2017).

[51] D. Barredo, V. Lienhard, S. de Léséleuc, T. Lahaye, and A. Browaeys, Nature 561, 79 (2018).

[52] T. D. Lee, F. E. Low, and D. Pines, Phys. Rev. 90, 297 (1953), URL https://link.aps.org/doi/10.1103/ PhysRev .90.297.

[53] D. Barredo, V. Lienhard, P. Scholl, S. de Léséleuc, T. Boulier, A. Browaeys, and T. Lahaye, Phys. Rev. Lett. 124, 023201 (2020), URL https://link.aps.org/doi/ 10.1103/PhysRevLett.124.023201.

[54] L. Landau and E. Lifshitz, Statistical Physics, v. 5 (Elsevier Science, 1980), ISBN 9780750633727, URL https: //books.google.de/books?id=dEVtKQEACAAJ.

[55] P. W. Anderson, Phys. Rev. Lett. 18, 1049 (1967), URL https://link.aps.org/doi/10.1103/PhysRevLett.18. 1049.

[56] J. Sous and M. Pretko, arXiv e-prints arXiv:1904.08424 (2019), 1904.08424.

[57] X.-F. Shi, Phys. Rev. Applied 13, 024008 (2020), URL https://link.aps.org/doi/10.1103/PhysRevApplied. 13.024008 .

[58] Y. Sun, P. Xu, P.-X. Chen, and L. Liu, Phys. Rev. Applied 13, 024059 (2020), URL https://link.aps.org/doi/10. 1103/PhysRevApplied.13.024059.

[59] Note, that these two quantities can be tuned independently, as shown in [47]. 